\title{
Experiences Migrating Microcosm Learning Materials
}

\author{
H.C. Davis \\ Learning Technology Group, ECS \\ The University of Southampton \\ Southampton, UK \\ +442380593669 \\ hcd@ecs.soton.ac.uk
}

\begin{abstract}
Microcosm was an open hypertext system that evolved in the early 1990s, before the advent of the Web. Apart from its success as a research platform it was widely used for presenting interactive educational materials. Since the commercial version of the product ceased to be supported it became necessary for users to migrate their educational materials, generally to the Web. However, the SToMP consortium chose to implement their own environment copying parts of the functionality of Microcosm in order to achieve their educational objectives. This paper examines the motivations of this work in order to understand whether there were features that were available in Microcosm that were not replicated in current Web based solutions.
\end{abstract}

\section{Categories and Subject Descriptors K.3.1 [Computer Uses in Education]}

\section{General Terms: Design, Human Factors}

Keywords: Microcosm, Authoring, Hypertext for Education

\section{INTRODUCTION}

The Microcosm open hypertext system [1] was developed in the late 80 's and early 90's. Its modular message passing design made it very flexible, and many students and research projects contributed to its comprehensive range of features. In spite of much academic interest in the ideas that the system embodied, and a financially successful embodiment as a commercial product, the user base did not expand much after the early adopters. There may be many reasons for this [2] but certainly the most important was the advent of the Web which conclusively demonstrated that the most important feature of any hypertext system was unfettered and non platform specific global access to documents, in spite of the fact that the hypertext features of the Web were inferior to most of the research-based systems of the ' $90 \mathrm{~s}$.

By the year 2000, however it had become clear that the Web infrastructure was open and could be programmed to behave as required; e.g see [3] for details of various approaches that have been taken to incrementing the basic Web model of the link. By the end of the 1990's most authors who had created hypertexts in earlier non-Web based systems needed to migrate their creations

Permission to make digital or hard copies of all or part of this work for personal or classroom use is granted without fee provided that copies are not made or distributed for profit or commercial advantage and that copies bear this notice and the full citation on the first page. To copy otherwise, or republish, to post on servers or to redistribute to lists, requires prior specific permission and/or a fee.

HT'04, August 9-13, 2004, Santa Cruz, California, USA.

Copyright 2004 ACM 1-58113-848-2/04/0008...\$5.00.

\author{
R.A. Bacon \\ Department of Physics \\ University of Surrey \\ Guildford, UK \\ +441483689414 \\ r.bacon@surrey.ac.uk
}

as they ceased to work with new versions of operating systems.

Teachers had been attracted to using Microcosm by the ease and freedom of authoring and the ability to present materials in pedagogically interesting ways [1]. One such project that had used Microcosm was SToMP [4] - a consortium of UK Universities founded in 1992 with HE Funding Council backing, to create electronic resources for the teaching of Physics. This paper examines the experiences of the SToMP team in migrating their materials to reflect on what functionality might still be missing from current Web based learning environments.

\section{MICROCOSM FEATURES IN STOMP}

The style of the SToMP system has the main "lessons" as rich text documents with links to the other documents authored as components of the lesson. These are typically simulations, animations, pictures, diagrams and video clips. A large amount of linked background information is available in the form of a glossary, a databook, biographies, and foundation units. The four SToMP modules contained a total of about 3500 documents including over 500 programmed animations and simulations written in a range of languages. These simulations and animations are central to the modules, providing essential insight to the concepts being developed in each lesson. These applications were written to be able to communicate with the Microcosm link service so that, for example, a student could ask a query or follow links from terms used within the simulation.

The SToMP system makes extensive use of all the Microcosm link types. Buttons are used as structural links e.g. from the main "lesson" to sub parts of the lesson. Generic links are used extensively as reference links; a large range of terms have been linked to their definitions in a glossary - so many in fact that nearly every technical word in the texts is linked. Local links have been used to provide associative links within a particular source document context, and SToMP makes use of document-todocument links to point readers to related documents. In addition computed links (content based queries using Latent Semantic Indexing techniques) were available.

A feature of Microcosm linking was that link information was held in a linkbase and not in the documents themselves. SToMP took advantage of this by preparing two separate versions of a module, one for conventional use by students, the other for use by academics in lecture situations, where they would want to link to the animations and simulations for demonstration purposes. The SToMP system also made use of the Microcosm API, adding its own 'floating toolbar' that provided button access to the Microcosm features as well as to a Physicist's toolkit, and creating a 'keyword browser' feature. 


\section{MIGRATING SToMP}

The SToMP team initially considered migrating the system to the Web, using Web browsers as viewers and they carried out a pilot study to test the feasibility. It was found, however, that creating a system that closely matched the look and feel of the original SToMP/Microcosm system would prove very time consuming. The principal difficulties encountered were the estimated amount of work required to convert the hundreds of formatted text files, many containing equations, to satisfactorily rendered html files, and the technical difficulties involved with developing an environment for the interactive programs so that they could be installed and executed on the client machines, but could communicate with the system. Other problematic issues included the lack of control of the appearance of text pages and the anticipated maintenance problems with the multiplicity of browsers and other third party support software used.

There is little doubt that these problems could eventually be solved (although it was anticipated that considerable compromise on the functionality and appearance would be required), but it was beyond the resources of the project to undertake the work. Rather than migrating to the Web the SToMP team chose to reimplement the components of Microcosm that they considered important using a Delphi application (downloadable from their Website) that provided the toolbar interface and access over the Internet to an SQL database holding the documents, links, simulations, animations and the remaining run-time components. Users may elect to use a version which locally caches objects when they have been downloaded once, thus reducing Internet accesses. The team believes that this implementation gives them all the advantages of the features and user interface of Microcosm that they need, while allowing them to maintain and update a single program and data source on the Internet.

Microcosm was the result of many years of development and the reader might reasonably assume that it would be unthinkable to re-implement instead of converting the resources. But in practice it was not necessary to re-implement it all, as modern $\mathrm{OO}$ development environments provide much higher level features (such as a rich text component) which can simply be extended to provide features that previously took significant development. Perhaps more importantly we should observe that Microcosm had a general purpose extensible architecture designed for multiple purposes, whereas the SToMP team had a very tight specification of the features they would be using, so it was not necessary for them to implement features like the tagged message format or filter service architecture.

\section{CONCLUSIONS}

Clearly some of the issues encountered by the SToMP team are to do with the cost of conversion of materials from one system to another; we have a case of "if I had wanted to go there I would not have started here". But the purpose of this paper is to reflect on this migration experience to see what it was that motivated the SToMP team to take this apparently radical approach rather than follow the more obvious path to the open Web (as provided by standard Web servers and browsers) or to current Web based virtual learning environments (VLEs).

On the face of it the Web seemed the obvious solution. It can be extended, e.g. by a link service on the server or by the use of browser plugins to render mathematical equations, but the task of harnessing the full range of tools needed to replicate the
Microcosm functionality was seen as both difficult and fragile, as was the task of either re-implementing or re-interfacing some hundreds of Windows based simulations. The SToMP team preferred a solution where all students would be using the same tool set. The pedagogic tools provided with current VLEs such as the on-line test engines, discussion boards, etc. and the class management tools might have been seen as powerful reasons for a VLE based re-implementation. In practice, however, most of these tools were not relevant to the SToMP style, where the pedagogic model is based on each student learning by exploring and experimenting with a set of professionally pre-prepared resources.

The above perhaps goes some way to explaining the reasons why the SToMP team did not wish to use a Web or VLE solution. But what is it about Microcosm that the team particularly wanted to retain? The answer is fourfold: they found that they wanted a) to reproduce the tight control of the way documents could be displayed in Microcosm using multiple windows, that cannot easily be achieved using general purpose browsers, b) to continue using the rich linking features that were provided with the Microcosm system, with the multiplicity of link types and the ability to link from the existing simulations as well as from conventional documents, c) to be able to continue using an authoring environment in which large numbers of different types of link can be created and maintained easily, d) to be able to continue using a graphical interface to organise documents (and reorganise them without breaking links).

The SToMP team have taken advantage of owning their own implementation to extend the document-to-document link type, to improve the Guided Path feature, and are currently adding stemming to generic link source selections so that words such as "computer" and "computers" will resolve to the same link.

The lesson we learn from this observation is that authoring for specialist applications often requires specialist tools. Another good example of this is in the writing community where Storyspace [5] is still much used by authors, even if the eventual delivery environment is to be the Web. The reason that VLEs have been so popular in certain areas is that they generally provide the toolkit that authors want. The SToMP project, however, is an example of where the specialist requirements were more important, and a re-implementation of Microcosm was the most cost effective way of achieving the required functionality

\section{References}

[1] Hall, W., Davis, H.C., \& Hutchings, G.A. Rethinking Hypermedia: The Microcosm Approach. Kluwer. 1996

[2] Davis, H.C. \& White, S.A. Linking Experiences: Issues Raised Developing Linkservices for Resource Based Learning and Teaching. In Proc IEEE Conf. on Advanced Learning Technologies: ICALT, Madison, 2001

[3] Bouvin, N.O., Unifying strategies for Web augmentation. Proc 10th ACM Conf. on Hypertext and hypermedia. 1999

[4] Bacon, R.A. \& Swithenby, S.J. A strategy for the integration of IT-led methods into physics - the SToMP approach. Computers \& Education, 26 pp135-141 1996.

[5] Bernstein, M. Storyspace 1, In Proc 13th ACM Conf. on Hypertext and Hypermedia, Maryland, USA, ACM 2002 